\title{
A simple method for improving the properties of the sago starch films prepared by using ultrasonication treatment
}

\begin{abstract}
Starch granules containing amylopectin-rich fractions like sago starch may remain insoluble and undamaged decreasing properties of the film. The aim of this study is to characterize native sago starch films prepared using ultrasonication. An ultrasonication probe was used during gelatinization for $2.5,5$, and 10 min respectively. Ultrasonication decreases the incomplete gelatinized granules resulting in a film with a more compact structure, and lower moisture vapor permeability than non-treated film. The longest duration resulted in a film with the highest transparency, and the highest thermal resistance. The duration for 5 min increased tensile strength of the film by $227 \%$, and its moisture absorption decreased by $29.83 \%$ compared to non-sonicated film. After ultrasonication for $10 \mathrm{~min}$, melting temperature increased by $7 \%$ in comparison to non-sonicated film. This work promotes a simple method to improve the tensile and physical properties of starch based film.
\end{abstract}

Keyword: Starch film; Transparency; Ultrasonication; Ghost 